Proceedings of the 2011 Winter Simulation Conference

S. Jain, R. R. Creasey, J. Himmelspach, K. P. White, and M. Fu, eds.

\title{
ON THE MEAN-SQUARED ERROR OF VARIANCE ESTIMATORS FOR COMPUTER SIMULATIONS
}

\author{
Tûba Aktaran-Kalaycı \\ Risk Analytics Department \\ SunTrust Bank \\ Atlanta, GA 30308, USA \\ David Goldsman \\ H. Milton Stewart School of \\ Industrial and Systems Engineering \\ Georgia Institute of Technology \\ Atlanta, GA 30332, USA
}

Christos Alexopoulos

H. Milton Stewart School of

Industrial and Systems Engineering

Georgia Institute of Technology

Atlanta, GA 30332, USA

James R. Wilson

Edward P. Fitts Department of

Industrial and Systems Engineering

North Carolina State University

Raleigh, NC 27695-7906, USA

\begin{abstract}
Given an output process generated by a steady-state simulation, we give expressions for the mean-squared error (MSE) of several well-known estimators of the associated variance parameter. The variance estimators are based on the method of nonoverlapping batch means and on the method of standardized time series applied to overlapping batch means. Under certain conditions, the resulting expressions are used to minimize the MSE with respect to the batch size, where the optimal batch size is expressed as a function of the simulation run length and certain moment properties of the output process. The ultimate objective is to exploit these results to construct new variance estimators with improved accuracy and efficiency, and to provide useful guidelines on setting the batch size in practice.
\end{abstract}

\section{INTRODUCTION}

An important problem in steady-state simulation output analysis involves the analysis of estimators associated with the mean. In particular, suppose that $\left\{Y_{1}, Y_{2}, \ldots\right\}$ is the output process generated by a discrete-event simulation; and further suppose that this process is stationary with steady-state mean $\mu=\mathrm{E}\left[Y_{i}\right]$ for $i=1,2, \ldots$. Examples of such processes include the following: (a) a Markov chain whose initial condition is sampled from the chain's steady-state distribution; (b) a Markov chain with an arbitrary initial condition that has been have been sufficiently warmed up; and (c) successive cycle times for entities flowing through a large queueing-network model of a production or communication system that has been sufficiently warmed up. The tried-and-true estimator for $\mu$ is obviously the sample mean based on $n$ consecutive observations, $\bar{Y}_{n} \equiv n^{-1} \sum_{i=1}^{n} Y_{i}$. Analysts might also be well-served to provide an estimator for $\operatorname{Var}\left(\bar{Y}_{n}\right)$, or almost equivalently, $\sigma^{2} \equiv \lim _{n \rightarrow \infty} n \operatorname{Var}\left(\bar{Y}_{n}\right)$. The quantity $\sigma^{2}$ is referred to as the asymptotic variance parameter and, assuming it exists, is useful in making precision and confidence statements about the sample mean as a point estimator for the unknown steady-state mean $\mu$. This paper studies the performance of estimators for $\sigma^{2}$ with attention given to their bias, variance, and mean-squared error (MSE). Our emphasis will be on estimators that are based on what have come to be known as the methods of nonoverlapping batch means (NBM) (Schmeiser 1982), overlapping batch means (OBM) (Meketon and Schmeiser 1984), and standardized time series (STS) (Schruben 1983). 


\section{Aktaran-Kalaycı, Alexopoulos, Goldsman, and Wilson}

This article is organized as follows. We present background material in Section 2, mainly consisting of a description of certain standard variance estimators that will be used to push the discussion forward. The main MSE results for more-general variance estimators are given in Section 3. The slides for the oral presentation of this article are available online via www.ise.ncsu.edu/jwilson/files/wsc11 mse.pdf [accessed October 25, 2011].

\section{BACKGROUND}

The goal in this section is to set the stage for some general results by first discussing some specific variance estimators that have found some popularity in the steady-state simulation output analysis literature namely, the NBM, OBM, and STS area estimators. These estimators all employ the use of batching, as will be explained below. We discuss generalizations of these results to other estimators in Section 3 .

\subsection{Nonoverlapping Batch Means Estimator}

Suppose we break the steady-state simulation-generated output $\left\{Y_{j}: j=1,2, \ldots, n\right\}$ of length $n$ into $b$ contiguous, nonoverlapping batches of observations, each of length $m$, where we assume for ease of exposition that $n=b m$. Thus, the observations $\left\{Y_{(i-1) m+k}: k=1,2, \ldots, m\right\}$ constitute the $i$ th batch for $i=1,2, \ldots, b$. In the sequel, we always take $b \equiv n / m$ so that $b$ always represents the ratio of the sample size to the batch size; and when we work with nonoverlapping batches as in this subsection, $b$ also equals the number of batches.

The quantities $\bar{Y}_{i, m} \equiv m^{-1} \sum_{k=1}^{m} Y_{(i-1) m+k}, i=1,2, \ldots, b$, are the nonoverlapping batch means; and under mild moment and mixing conditions, these batch means become approximately i.i.d. normal random variables as the batch size $m$ increases. This justifies the use of the scaled sample variance of the batch means as the NBM estimator for $\sigma^{2}$ (Glynn and Whitt 1991, Steiger and Wilson 2001),

$$
\mathscr{N}(b, m) \equiv \frac{m}{b-1} \sum_{i=1}^{b}\left(\bar{Y}_{i, m}-\bar{Y}_{n}\right)^{2} \Rightarrow \frac{\sigma^{2} \chi_{b-1}^{2}}{b-1},
$$

where the symbol $\Rightarrow$ denotes convergence in distribution as $m \rightarrow \infty$, and $\chi_{v}^{2}$ is a $\chi^{2}$ random variable with $v$ degrees of freedom. Under mild conditions, several papers (e.g., Chien, Goldsman, and Melamed 1997, Goldsman and Meketon 1986, Song and Schmeiser 1995) show that the expected value of the NBM estimator is of the form

$$
\mathrm{E}[\mathscr{N}(b, m)]=\sigma^{2}-\frac{\gamma_{1}(b+1)}{m b}+O\left(1 / m^{2}\right),
$$

where: (a) the "Big-Oh" notation $g(m)=O(h(m))$ means that for some finite constants $C$ and $m_{0}$, we have $|g(m)| \leq C|h(m)|$ for all $m \geq m_{0}$; and (b) the constants

$$
\gamma_{\ell} \equiv 2 \sum_{k=1}^{\infty} k^{\ell} \operatorname{Cov}\left[Y_{1}, Y_{1+k}\right] \quad \text { for } \ell=1,2, \ldots
$$

provide a convenient characterization of the covariance structure of the process $\left\{Y_{j}: j=1,2, \ldots\right\}$. Similarly, the NBM estimator's variance is given by

$$
\lim _{m \rightarrow \infty}(b-1) \operatorname{Var}[\mathscr{N}(b, m)]=2 \sigma^{4} \quad \text { for fixed } b .
$$

\subsection{Overlapping Batch Means Estimator}

Now we form $n-m+1$ overlapping batches, each of size $m$, from $\left\{Y_{j}: j=1,2, \ldots, n\right\}$. Specifically, the $i$ th overlapping batch is composed of the observations $\left\{Y_{i+k}: k=0, \ldots, m-1\right\}$, for $i=1,2, \ldots, n-m+1$; the $i$ th overlapping batch mean is $\bar{Y}_{i, m}^{\mathrm{O}} \equiv \sum_{k=0}^{m-1} Y_{i+k} / m$, for $i=1,2, \ldots, n-m+1$. The OBM estimator for 


\section{Aktaran-Kalaycı, Alexopoulos, Goldsman, and Wilson}

$\sigma^{2}$ is the scaled sample variance of the (highly correlated) overlapping batch means (cf. Meketon and Schmeiser 1984),

$$
\mathscr{O}(b, m) \equiv \frac{n m}{(n-m+1)(n-m)} \sum_{i=1}^{n-m+1}\left(\bar{Y}_{i, m}^{\mathrm{O}}-\bar{Y}_{n}\right)^{2} .
$$

Under mild conditions, Goldsman and Meketon (1986) and Song and Schmeiser (1995) (among others) show that

$$
\mathrm{E}[\mathscr{O}(b, m)]=\sigma^{2}+\frac{\gamma_{1}\left(b^{2}+1\right)}{m b(b-1)}+O\left(1 / m^{2}\right) .
$$

Further, following the lead of Meketon and Schmeiser (1984), Damerdji (1995) finds that for large batch size $m$ and sample-to-batch-size ratio $b$,

$$
\lim _{m \rightarrow \infty} \operatorname{Var}[\mathscr{O}(b, m)]=\frac{\left(4 b^{3}-11 b^{2}+4 b+6\right) \sigma^{4}}{3(b-1)^{4}} \sim \frac{4 \sigma^{4}}{3 b} \quad \text { as } b \rightarrow \infty .
$$

The OBM estimator has about the same bias as, but only $2 / 3$ the variance of the NBM estimator.

\subsection{Standardized Time Series Estimators}

Schruben (1983) defined the standardized time series from nonoverlapping batch $i$ by

$$
T_{i, m}(t) \equiv \frac{\lfloor m t\rfloor\left(\bar{Y}_{i, m}-\bar{Y}_{i,\lfloor m t\rfloor}\right)}{\sigma \sqrt{m}} \quad \text { for } t \in[0,1] \text { and } i=1,2, \ldots, b
$$

where $\lfloor\cdot\rfloor$ is the floor function and $\bar{Y}_{i, j} \equiv j^{-1} \sum_{k=1}^{j} Y_{(i-1) m+k}$ is the $j$ th cumulative sample mean from batch $i$, for $i=1,2, \ldots, b$ and $j=1,2, \ldots, m$. Schruben then proposed what is known as the area estimator for $\sigma^{2}$, which corresponds to the squared area under the STS from each batch,

$$
\mathscr{A}(f ; b, m) \equiv \frac{1}{b} \sum_{i=1}^{b} A_{i}(f ; m),
$$

where

$$
A_{i}(f ; m) \equiv\left[\frac{1}{m} \sum_{k=1}^{m} f(k / m) \sigma T_{i, m}(k / m)\right]^{2} \quad \text { for } i=1,2, \ldots, b,
$$

and where the weight function $f(\cdot)$ satisfies the conditions

$$
\int_{0}^{1} \int_{0}^{1} f(s) f(t)(\min (s, t)-s t) d s d t=1 \quad \text { and } \quad \frac{d^{2}}{d t^{2}} f(t) \text { is continuous at every } t \in[0,1] .
$$

Under a mild functional central limit theorem assumption (cf. Alexopoulos et al. 2007), it turns out that $\mathscr{A}(f ; b, m) \Rightarrow \sigma^{2} \chi_{b}^{2} / b$.

In addition, Goldsman, Meketon, and Schruben (1990) show that

$$
\mathrm{E}[\mathscr{A}(f ; b, m)]=\sigma^{2}-\frac{\left[(F-\bar{F})^{2}+\bar{F}^{2}\right] \gamma_{1}}{2 m}+O\left(1 / m^{2}\right),
$$

where

$$
F(s) \equiv \int_{0}^{s} f(t) d t \text { for } s \in[0,1], F \equiv F(1), \bar{F}(u) \equiv \int_{0}^{u} F(s) d s \text { for } u \in[0,1], \text { and } \bar{F} \equiv \bar{F}(1) .
$$


Further, under mild conditions, we have

$$
\lim _{m \rightarrow \infty} b \operatorname{Var}[\mathscr{A}(f ; b, m)]=2 \sigma^{4} .
$$

What makes the STS results particularly interesting is that there is a great deal of flexibility on the choice of weight functions that satisfy Condition (6). For instance, Schruben's original area estimator used the constant weight $f_{0}(t) \equiv \sqrt{12}$ for all $t \in[0,1]$, for which Equation (7) yields $\mathrm{E}\left[\mathscr{A}\left(f_{0} ; b, m\right)\right]=$ $\sigma^{2}-3 \gamma_{1} / m+O\left(1 / m^{2}\right)$. A nice consequence of the flexibility of (6) is that it is easy to choose a weight such that $F=\bar{F}=0$, which results in an estimator that is first-order unbiased for $\sigma^{2}$. For instance, for the weight $f_{2}(t) \equiv \sqrt{840}\left(3 t^{2}-3 t+1 / 2\right)$, Aktaran-Kalayc1 et al. (2007) derive the fine-tuned result

$$
\mathrm{E}\left[\mathscr{A}\left(f_{2} ; b, m\right)\right]=\sigma^{2}+\frac{7\left(\sigma^{2}-6 \gamma_{2}\right)}{2 m^{2}}+O\left(1 / m^{3}\right)
$$

\section{MAIN RESULTS}

We now consider a generic variance estimator $\widehat{V}$ for the variance parameter $\sigma^{2}$. Suppose that the bias of $\widehat{V}$ is of the form $\operatorname{Bias}(\widehat{V})=c / m^{k}$ for some constant $c$, batch size $m$, and $k>0$, where we continue to ignore smaller-order terms. Further suppose that the variance of $\widehat{V}$ is of the form $\operatorname{Var}(\widehat{V})=v / b$ for some constant $v$ and sample-to-batch-size ratio $b=n / m$. Table 1, which summarizes Equations (1)-(9), shows that the bias and variance results for the NBM, OBM, and STS area estimators adhere to the assumed forms. In fact, a number of other variance estimators from the literature have these forms of bias and variance; see Section 4.

Table 1: Approximate bias and variance for different estimators of $\sigma^{2}$.

\begin{tabular}{ccc}
\hline Estimator & Bias & Variance \\
\hline $\mathscr{N}(b, m)$ & $\frac{\gamma_{1}(b+1)}{m b}$ & $\frac{2 \sigma^{4}}{b-1}$ \\
$\mathscr{O}(b, m)$ & $\frac{\gamma_{1}\left(b^{2}+1\right)}{m b(b-1)}$ & $\frac{\left(4 b^{3}-11 b^{2}+4 b+6\right) \sigma^{4}}{3(b-1)^{4}}$ \\
$\mathscr{A}\left(f_{0} ; b, m\right)$ & $\frac{3 \gamma_{1}}{m}$ & $\frac{2 \sigma^{4}}{b}$ \\
$\mathscr{A}\left(f_{2} ; b, m\right)$ & $\frac{7\left(\sigma^{2}-6 \gamma_{2}\right)}{2 m^{2}}$ & $\frac{2 \sigma^{4}}{b}$ \\
\hline
\end{tabular}

In such cases, the MSE of $\widehat{V}$ as an estimator of $\sigma^{2}$ is

$$
\operatorname{MSE}(\widehat{V})=\operatorname{Bias}^{2}(\widehat{V})+\operatorname{Var}(\widehat{V})=\frac{c^{2}}{m^{2 k}}+\frac{v}{b} .
$$

Following the lead of Goldsman and Meketon (1986) and Song and Schmeiser (1995), we will minimize this quantity (at least asymptotically for large values of the run length $n$ and hence for large $m=m(n)$ and $b=b(n)$ ). To do so, we take $b(n)=\alpha n^{\delta}$ and $m(n)=n^{1-\delta} / \alpha$, for some appropriately chosen parameters $\alpha>0$ and $0<\delta<1$; and with this parameterization, we have

$$
\operatorname{MSE}(\widehat{V}) \sim \frac{\alpha^{2 k} c^{2}}{n^{(1-\delta) 2 k}}+\frac{v}{\alpha n^{\delta}} \text { as } n \rightarrow \infty
$$




\section{Aktaran-Kalaycı, Alexopoulos, Goldsman, and Wilson}

where the symbol $\sim$ means "is asymptotic to" so that the ratio of the left-hand side of (11) to its right-hand side approaches one as $n \rightarrow \infty$.

The following heuristic argument motivates our formulation of the batch size $m^{\star}=m^{\star}(n)$ that minimizes $\operatorname{MSE}(\widehat{V})$ for large $n$. Differentiating the right-hand side of (11) with respect to $\delta$, setting the result equal to zero, and rearranging terms, we have the relation

$$
n^{2 k-\delta(2 k+1)} \sim \frac{2 k \alpha^{2 k+1} c^{2}}{v} \text { as } n \rightarrow \infty .
$$

Because the right-hand side of (12) does not depend on $n$, the exponent $2 k-\delta(2 k+1)$ of the run length $n$ on the left-hand side of (12) must be exactly zero, which in turn implies that the right-hand side of (12) must be exactly one; and thus for the asymptotically optimal values of $\delta$ and $\alpha$, we must have

$$
\delta^{\star}=\frac{2 k}{2 k+1} \quad \text { and } \quad \alpha^{\star}=\left(\frac{v}{2 k c^{2}}\right)^{1 /(2 k+1)} .
$$

Therefore we obtain the asymptotically optimal batch size of

$$
m^{\star}(n)=\frac{n^{1-\delta^{\star}}}{\alpha^{\star}}=\left(\frac{2 k c^{2} n}{v}\right)^{1 /(1+2 k)}
$$

and the asymptotically optimal sample-to-batch-size ratio of

$$
b^{\star}(n)=\alpha^{\star} n^{\delta^{\star}}=\left(\frac{v}{2 k c^{2}}\right)^{1 /(2 k+1)} n^{2 k /(2 k+1)} .
$$

Substituting the values of $m^{\star}(n)$ and $b^{\star}(n)$ into Equation (10) yields the asymptotically optimal MSE,

$$
\operatorname{MSE}^{\star}(\widehat{V})=\left(\frac{v^{k} c}{n^{k}}\right)^{\frac{2}{1+2 k}}\left[\left(\frac{1}{2 k}\right)^{\frac{2 k}{1+2 k}}+(2 k)^{\frac{1}{1+2 k}}\right]=(1+2 k)\left[c\left(\frac{v}{2 n k}\right)^{k}\right]^{\frac{2}{1+2 k}} .
$$

Table 2 gives approximate asymptotic optimality results for all the variance estimators considered in this article based on Equations (13) through (16). Notice that the OBM estimator's optimal MSE is lower than that of the NBM estimator, which in turn is lower than that of the STS area estimator with uniform weight function $f_{0}(\cdot)$ - results that are well known in light of Goldsman and Meketon (1986) and Song and Schmeiser (1995). For all three of these variance estimators, the optimal MSE declines as $n^{-2 / 3}$. By contrast, the optimal MSE of the STS area estimator with first-order unbiased weight function $f_{2}(\cdot)$ declines as $n^{-4 / 5}$; and this latter property strongly suggests that further significant improvements in the estimation of $\sigma^{2}$ are achievable.

Table 2: Approximate asymptotic bias and variance for different estimators.

\begin{tabular}{cccccc}
\hline Estimator & $k$ & $c$ & $v$ & $m^{\star}$ & $\operatorname{MSE}^{\star}(\widehat{V})$ \\
$\mathscr{N}(b, m)$ & 1 & $\gamma_{1}$ & $2 \sigma^{4}$ & $\left(\frac{\gamma_{1}^{2} n}{\sigma^{4}}\right)^{1 / 3}$ & $3\left(\frac{\gamma_{1} \sigma^{4}}{n}\right)^{2 / 3}$ \\
$\mathscr{O}(b, m)$ & 1 & $\gamma_{1}$ & $\frac{4 \sigma^{4}}{3}$ & $\left(\frac{3 \gamma_{1}^{2} n}{2 \sigma^{4}}\right)^{1 / 3}$ & $2.289\left(\frac{\gamma_{1} \sigma^{4}}{n}\right)^{2 / 3}$ \\
$\mathscr{A}\left(f_{0} ; b, m\right)$ & 1 & $3 \gamma_{1}$ & $2 \sigma^{4}$ & $\left(\frac{9 \gamma_{1}^{2} n}{\sigma^{4}}\right)^{1 / 3}$ & $6.240\left(\frac{\gamma_{1} \sigma^{4}}{n}\right)^{2 / 3}$ \\
$\mathscr{A}\left(f_{2} ; b, m\right)$ & 2 & $\frac{7\left(\sigma^{2}-6 \gamma_{2}\right)}{2}$ & $2 \sigma^{4}$ & {$\left[\frac{49\left(\sigma^{2}-6 \gamma_{2}\right)^{2} n}{2 \sigma^{4}}\right]^{1 / 5}$} & $4.740\left[\frac{\left(\sigma^{2}-6 \gamma_{2}\right) \sigma^{8}}{n^{2}}\right]^{2 / 5}$ \\
\hline
\end{tabular}




\section{AUGMENTATIONS AND CONCLUSIONS}

The discussion in the current paper has only considered a few basic estimators - NBM, OBM, and certain elementary STS area estimators. The idea was to show how to obtain optimal MSE expressions for a reasonably large class of estimators for $\sigma^{2}$; and to show that certain first-order unbiased estimators have optimal MSEs that are significantly better than their competitors. Of course, many other estimators fit into the MSE paradigm discussed herein, including additional STS area estimators (e.g., Foley and Goldsman 1999) and STS Cramér-von Mises estimators (Goldsman, Kang, and Seila 1999). In addition, we are currently investigating methods to produce minimum-MSE linear combinations of estimators for $\sigma^{2}$ (e.g., Aktaran-Kalayci et al. 2009).

One might notice that all of our expressions for the optimal batch size and optimal MSE involve quantities such as $\sigma^{2}$ and $\gamma_{\ell}$ that will undoubtedly be unknown in practice. Song (1996) developed methods for estimating the ubiquitous ratio $\gamma_{1}^{2} / \sigma^{4}$ for a variety of processes, including moving average processes and autoregressive processes, and Sherman (1995) proposed an alternative MSE-minimization strategy that does not rely on the estimation of $\gamma_{1}^{2} / \sigma^{4}$. Analogous technology will have to be developed to estimate other unknown quantities involving $\gamma_{\ell}$ and $\sigma^{2}$. In any case, when acceptable estimates can be obtained, then one can plug those estimates into the appropriate entries of Table 2.

\section{ACKNOWLEDGMENTS}

The work of Christos Alexopoulos was partially supported by National Science Foundation grant EFRI ARES-CI 0735991.

\section{REFERENCES}

Aktaran-Kalaycı, T., C. Alexopoulos, N. T. Argon, D. Goldsman, and J. R. Wilson. 2007. "Exact Expected Values of Variance Estimators in Steady-State Simulation.” Naval Research Logistics 54 (4): 397-410.

Aktaran-Kalaycı, T., C. Alexopoulos, D. Goldsman, and J. R. Wilson. 2009. "Optimal Linear Combinations of Overlapping Variance Estimators for Steady-State Simulation." In Advancing the Frontiers of Simulation: A Festschrift in Honor of George Samuel Fishman, edited by C. Alexopoulos, D. Goldsman, and J. R. Wilson, 291-328. New York: Springer Science+Business Media.

Alexopoulos, C., N. T. Argon, D. Goldsman, G. Tokol, and J. R. Wilson. 2007. "Overlapping Variance Estimators for Simulation.” Operations Research 55 (6): 1090-1103.

Chien, C.-H., D. Goldsman, and B. Melamed. 1997. "Large-Sample Results for Batch Means." Management Science 43:1288-1295.

Damerdji, H. 1995. "Mean-Square Consistency of the Variance Estimator in Steady-State Simulation Output Analysis." Operations Research 43 (2): 282-291.

Foley, R. D., and D. Goldsman. 1999. "Confidence Intervals Using Orthonormally Weighted Standardized Time Series." ACM Transactions on Modeling and Simulation 9:297-325.

Glynn, P. W., and W. Whitt. 1991. "Estimating the Asymptotic Variance with Batch Means." Operations Research Letters 10:431-435.

Goldsman, D., K. Kang, and A. F. Seila. 1999. "Cramér-von Mises Variance Estimators for Simulations." Operations Research 47:299-309.

Goldsman, D., and M. S. Meketon. 1986. "A Comparison of Several Variance Estimators.” Technical Report, School of Industrial and Systems Engineering, Georgia Institute of Technology, Atlanta, Georgia.

Goldsman, D., M. S. Meketon, and L. W. Schruben. 1990. "Properties of Standardized Time Series Weighted Area Variance Estimators." Management Science 36:602-612.

Meketon, M. S., and B. W. Schmeiser. 1984. "Overlapping Batch Means: Something For Nothing?" In Proceedings of the 1984 Winter Simulation Conference, edited by S. Sheppard, U. W. Pooch, and C. D. Pegden, 227-230. Piscataway, New Jersey: Institute of Electrical and Electronics Engineers, Inc. 
Schmeiser, B. W. 1982. "Batch Size Effects in the Analysis of Simulation Output." Operations Research 30:556-568.

Schruben, L. W. 1983. "Confidence Interval Estimation Using Standardized Time Series." Operations Research 31:1090-1108.

Sherman, M. 1995. "On Batch Means in the Simulation and Statistical Communities." In Proceedings of the 1995 Winter Simulation Conference, edited by C. Alexopoulos, K. Kang, W. R. Lilegdon, and D. Goldsman, 297-302. Piscataway, New Jersey: Institute of Electrical and Electronics Engineers, Inc.

Song, W.-M., and B. W. Schmeiser. 1995. "Optimal Mean-Squared-Error Batch Sizes." Management Science 41:110-123.

Song, W.-M. T. 1996. "On the Estimation of Optimal Batch Sizes in the Analysis of Simulation Output Analysis." European Journal of Operational Research 88:304-309.

Steiger, N. M., and J. R. Wilson. 2001. "Convergence Properties of the Batch-Means Method for Simulation Output Analysis.” INFORMS Journal on Computing 13 (4): 277-293.

\section{AUTHOR BIOGRAPHIES}

TÛBA AKTARAN-KALAYCI received her B.S. degree in Industrial Engineering from Bilkent University in Ankara, Turkey, and her Ph.D. from the H. Milton Stewart School of Industrial and Systems Engineering at the Georgia Institute of Technology. She is currently in the Risk Analytics Department at SunTrust Bank in Atlanta, Georgia. Her research interests lie in simulation output analysis and financial applications. Her e-mail address is taktaran@gmail.com.

CHRISTOS ALEXOPOULOS is an associate professor in the H. Milton Stewart School of Industrial and Systems Engineering at the Georgia Institute of Technology. His research interests are in the areas of simulation, statistics, and optimization of stochastic systems. He is a member of INFORMS and an active participant in the Winter Simulation Conference, having been Proceedings Co-Editor in 1995, Associate Program Chair in 2006, and a member of the Board of Directors since 2008. He is also an Area Editor of the ACM Transactions on Modeling and Computer Simulation. His e-mail address is christos@isye.gatech.edu, and his Web page is www.isye.gatech.edu/ christos.

DAVID GOLDSMAN is a professor in the H. Milton Stewart School of Industrial and Systems Engineering at the Georgia Institute of Technology. His research interests include simulation output analysis, ranking and selection, and healthcare simulation. He is a long-standing participant in the Winter Simulation Conference, having been Program Chair in 1995 and a member of the WSC Board of Directors between 2001-2009. His e-mail address is sman@gatech.edu, and his Web page is www.isye.gatech.edu/ $\sim \operatorname{sman}$.

JAMES R. WILSON is a professor in the Edward P. Fitts Department of Industrial and Systems Engineering at North Carolina State University. His current research interests are focused on probabilistic and statistical issues in the design and analysis of simulation experiments. He has held the following editorial positions: departmental editor of Management Science (1988-1996); area editor of ACM Transactions on Modeling and Computer Simulation (1997-2002); guest editor of a special issue of IIE Transactions honoring Alan Pritsker (1999-2001); and Editor-in-Chief of ACM Transactions on Modeling and Computer Simulation (2004-2010). He served The Institute of Management Sciences College on Simulation (now the INFORMS Simulation Society) as secretary-treasurer (1984-1986), vice president (1986-1988), and president (19881990). As a participant in the Winter Simulation Conference (WSC), he served as proceedings editor (1986), associate program chair (1991), and program chair (1992). As a member of the WSC Board of Directors corepresenting the INFORMS Simulation Society during the period 1997-2004, he served as secretary (2001), vice chair (2002), and chair (2003). He was a trustee of the WSC Foundation during the period 2006-2010. He is a member of ACM, ASA, and SCS; and he is a Fellow of IIE and INFORMS. His e-mail address is jwilson@ncsu.edu, and his Web page is www.ise.ncsu.edu/jwilson. 